\title{
Irinotecan, topotecan, paclitaxel or docetaxel for second-line treatment of small cell lung cancer: a single-center retrospective study of efficiency comparation and prognosis analysis
}

\author{
Yuan Zhao ${ }^{1,2 \#}$, Bing Wan ${ }^{3 \#}$, Tianli Zhang ${ }^{4}$, Yangyang Xu ${ }^{5}$, Hongbing Liu ${ }^{4,6}$, Tangfeng Lv ${ }^{4,6}$, Fang Zhang ${ }^{4,6}$, \\ Ping Zhan ${ }^{4,6}$, Yong Song ${ }^{4,6}$ \\ ${ }^{1}$ Department of Critical Care Medicine, School of Medicine, Zhongda Hospital, Southeast University, Nanjing 210000, China; ${ }^{2}$ Department \\ of Respiratory Medicine, Jinling Hospital, Nanjing Medical University, Nanjing 210002, China; ${ }^{3}$ Department of Respiratory and Critical Care \\ Medicine, The Affiliated Jiangning Hospital of Nanjing Medical University, Nanjing 210002, China; ${ }^{4}$ Department of Respiratory Medicine, Jinling \\ Hospital, Medical School of Southeast University, Nanjing 210000, China; ${ }^{5}$ Department of Respiratory Medicine, Jinling Hospital, Nanjing Medical \\ University, Nanjing 210000, China; ${ }^{6}$ Department of Respiratory Medicine, Jinling Hospital, Nanjing University School of Medicine, Nanjing \\ 210002, China \\ Contributions: (I) Conception and design: All authors; (II) Administrative support: Y Song, F Zhang, T Lv, H Liu; (III) Provision of study materials or \\ patients: Y Song, F Zhang, T Lv; (IV) Collection and assembly of data: All authors; (V) Data analysis and interpretation: Y Zhao, B Wan, P Zhan; (VI) \\ Manuscript writing: All authors; (VII) Final approval of manuscript: All authors. \\ \#These authors contributed equally to this work. \\ Correspondence to: Ping Zhan; Yong Song, MD, PhD. Department of Respiratory Medicine, Jinling Hospital, Nanjing University School of Medicine, \\ \#305, East Zhongshan Road, Nanjing 210002, China. Email: zhanping207@163.com; yong_song6310@yahoo.com.
}

Background: The main aim of this study was to evaluate the efficiency of second-line chemotherapy irinotecan (CPT-11), topotecan (TPT), paclitaxel (PTX) and docetaxel (DTX) in small cell lung cancer (SCLC) patients who have failure to the first-line standard treatment. The secondary aim was to evaluate the independent prognostic factors of patients who received a second line treatment.

Methods: Retrospective analysis of 116 patients who received second-line chemotherapy. Patients were divided into 4 groups according to the therapy they were treated with, which were CPT-11, TPT, PTX and DTX. Progress free survival (PFS), overall survival (OS), objective response rate (ORR) and disease control rate (DCR) were evaluated for each group. Patients' data of clinical character and blood index were collected, and the prognostic factors were assessed both at univariate and multivariate levels.

Results: Patients treated with CPT-11 achieved the best median PFS and OS of 91 and 595 days, while the median PFS of TPT, PTX and DTX were 74.5, 81 and 50 days respectively. The median OS of them were $154,168.5$ and 184 days respectively. The survival curves of OS were significantly different $(\mathrm{P}=0.0069)$. The reaction to second-line therapy is positively correlate to the reaction to first-line therapy $(\mathrm{P}=0.012)$. In the multivariate analysis, treatment free interval (TFI) $<90$ days, lactate dehydrogenase (LDH) $\geq 225 \mathrm{U} / \mathrm{L}$, neutrophil-to-lymphocyte ratio (NLR) $\geq 3.5$ were identified as independent risk factors for poor prognosis in second-line SCLC patients.

Conclusions: Second-line chemotherapy with TPT in SCLC patients may provide better overall survival benefits. TFI $<90$ days, $\mathrm{LDH} \geq 225 \mathrm{U} / \mathrm{L}$ and NLR $\geq 3.5$ are independent risk factors for second-line SCLC patients.

Keywords: Second-line therapy; small cell lung cancer (SCLC); topotecan; irinotecan; paclitaxel; docetaxel; prognostic factor

Submitted Jun 20, 2019. Accepted for publication Oct 12, 2019.

doi: $10.21037 /$ tlcr.2019.10.21

View this article at: http://dx.doi.org/10.21037/tlcr.2019.10.21 


\section{Introduction}

Small cell lung cancer (SCLC) is a highly malignant pulmonary tumor accounts for $10-15 \%$ of all lung cancers. It is quite aggressive with high doubling rate and priority of early distant metastasis and acquired drug resistance $(1,2)$. SCLC is highly sensitive to first-line treatment, the response rate (RR) of limited SCLC can reach 70-90\%, extensive SCLC can reach 50-60\%. However, about $80 \%$ of patients with limited stage and almost all patients with extensive stage will develop disease relapse or progression within 1 year of initial treatment (3-5). Patients need to receive appropriate second-line therapy after they progressed from the first-line chemotherapy.

For those who progressed from first-line chemotherapy, clinical trial enrollment is recommended by the National Comprehensive Cancer Network (NCCN) SCLC guidelines. There are few studies on the effectiveness and toxicity of SCLC second-line chemotherapy. To date, topotecan (TPT) is the best recommended therapy for second-line treatment of SCLC in Europe and the United States, while amrubicin (AMR), irinotecan (CPT-11), paclitaxel (PTX), docetaxel (DXL) and other drugs such as two immunotherapy agents (nivolumab and ipilimumab) have also shown some therapeutic effects on relapsed SCLC in some clinical studies (6-11). However, there is no standard second-line treatment plans and large-scale prospective test results for reference currently.

This retrospective study was conducted to assess the efficiency of second-line treatment in SCLC patients who have recurrence or progression after the first-line standard treatment. We analyzed the performance of four most commonly used second-line chemotherapy TPT, CPT-11, PTX and DXL in real-world clinical cases of patients with SCLC to provide a reference for clinical treatment. Beside of the efficiency comparation, we performed a multivariate analysis based the data of patients' clinical characters and serum tumor markers in order to find out the prognostic factors of SCLC patients who received second-line chemotherapy.

\section{Methods}

\section{Patients and treatment}

This retrospective study selected SCLC patients admitted to Jinling Hospital for medical treatment from January 2008 to December 2017. For this study, the eligibility criteria were as follows: histologically or cytologically confirmed SCLC; received standard first-line chemotherapy of etoposide plus platinum (cisplatin or carboplatin); were systematically divided into extensive disease (ED) and limited disease (LD) according to the criteria of Veterans Administration Lung Study Group (VALG) before treatment (12).

All patients were treated with standard first-line chemotherapy of etoposide plus platinum (cisplatin or carboplatin). Patients were divided into 4 groups according to the second-line chemotherapy they were treated with: CPT-11, TPT, PTX and DTX. Patients in these 4 groups were treated with single drug or with combine platinum (cisplatin or carboplatin). Blood index data of patients before treatment were collected.

This study is a retrospective study, approved by the ethics committee of Nanjing Jinling Hospital, without the need for informed consent.

\section{Evaluation}

The evaluation of tumor response to chemotherapy was conducted according to the Response Evaluation Criteria in Solid Tumors. Objective response rate (ORR) refers to the rate of patients got complete response (CR) and partial response (PR) while disease control rate (DCR) contains $\mathrm{CR}, \mathrm{PR}$ and stable disease (SD). Treatment free interval (TFI) was measured from the date of last first-line treatment to disease progression or the date of receiving subsequent therapy. Clinical evaluation of progress free survival (PFS) and overall survival (OS) was conducted by Kaplan-Meier method to assess the time from second-line chemotherapy to recurrence or death.

\section{Statistical analysis}

Chi-square test was used for qualitative data and the unqualified data were only displayed as frequency. Survival curves were depicted using Kaplan-Meier method by GraphPad Prism 7.00. Prognostic factors were assessed both at univariate and multivariate levels. Univariate analysis and multivariate analysis were performed by log-rank analysis and the Cox-regression model. All the statistical analyses were performed by GraphPad Prism 7.00 and SPSS version 22.0 (IBM Corp, Armonk, NY, USA). P values $<0.05$ were considered significant.

\section{Results}

\section{Patient characteristics}

From January 2008 to December 2017, 116 patients who 
Table 1 Clinical character of patients received first and second-line therapy

\begin{tabular}{|c|c|c|c|}
\hline Clinical characteristics & $\begin{array}{l}\text { First-line } \\
\text { therapy }\end{array}$ & $\begin{array}{l}\text { Second-line } \\
\text { therapy }\end{array}$ & $P$ \\
\hline Age & & & 0.816 \\
\hline$\leq 65$ & 180 & 75 & \\
\hline$>65$ & 92 & 41 & \\
\hline Gender & & & 0.744 \\
\hline Male & 231 & 100 & \\
\hline Female & 41 & 16 & \\
\hline Smoking & & & 0.964 \\
\hline Smoker & 202 & 86 & \\
\hline Non-smoker & 65 & 28 & \\
\hline Unknown & 5 & 2 & \\
\hline Stage at diagnosis & & & 0.81 \\
\hline Limited & 101 & 45 & \\
\hline Extensive & 166 & 70 & \\
\hline Unknown & 5 & 1 & \\
\hline Location of metastases & & & 0.529 \\
\hline Brain & 36 & 12 & \\
\hline Liver & 28 & 11 & \\
\hline Bone & 70 & 26 & \\
\hline Adrenal glands & 9 & 7 & \\
\hline
\end{tabular}

received second-line therapy after recurrence or progression from the first-line standard chemotherapy of etoposide plus platinum were included in this retrospective cohort study. The patients' clinical characters are shown in Table 1. There were 41 patients elder than 65 years old, 100 men and 16 women, 86 patients had a history of smoking, 45 patients in limited stage and 70 in extensive stage at the time of diagnose.

Table 2 shows the second-line treatment received by these patients. The number of patients received CPT-11, TPT, PTX and DTX were 28, 22, 25 and 34 respectively. Seven patients received other different second-line therapy were not included in further analysis because the cohorts were too small. There were no differences in baseline characteristics of age, smoking status, stage of disease and TFI among the four groups of patients. All 25 patients in PTX group were male and been treated with platinum at second-line therapy. The proportion of patients in limited stage and patients with longer TFI was higher in the CPT11 group (17/28 and 16/28) than the others, but there was no statistical difference.

\section{Response}

The response was evaluated in patients with complete medical information. The total ORR and DCR of patients who received second-line therapy was $19.05 \%$ and $61.90 \%$. For patients in different groups, the ORR of CPT-11, TPT, PTX and DTX were $22.22 \%, 15.38 \%, 21.43 \%$ and $16.67 \%$ respectively. The DCR of them were $66.7 \%$, $53.85 \%, 78.57 \%$ and $44.44 \%$ respectively (Figure 1). There was only one patient achieved CR, who was in the CPT-11 group and had achieved CR in the first-line therapy before. We made a correlation analysis between the reaction to second-line therapy and the reaction to first-line therapy, found that the two were positively correlated $(\mathrm{P}=0.012$, Table 3).

\section{Survival}

The survival data of patients who received different secondline therapy was analyzed by K-M curves. PFS and OS were determined from the time of second-line therapy. The median PFS of CPT-11, TPT, PTX and DTX were 91, 74.5, 81 and 50 days respectively (Figure $2 A$ ). Patients who used CPT-11 had the longest PFS, but there was no significant difference $(\mathrm{P}=0.6445)$. The median $\mathrm{OS}$ of them were 595, 154, 168.5 and 184 days respectively (Figure 2B), Patients who used CPT-11 had significantly longer OS than the other three groups $(\mathrm{P}=0.0069)$.

The OS of patients with different recurrent patterns was shown in Figure 3. According to the length of TFI, patients were divided into two groups with the boundary of 90 days. Patients whose TFI was 90 days and longer had significantly longer OS than the patient with TFI shorter than 90 days $(\mathrm{P}=0.0105)$. The medium OS of them were 288 and 172.5 days respectively.

\section{Prognostic factor analysis}

In order to find out which clinical characteristics and blood index are concerned with the survival of SCLC patients who received second-line chemotherapy, we performed the univariate and multivariate analysis for OS, which was summarized in Table 4. The cut-off value for PLT, LDH, neutrophil-to-lymphocyte ratio (NLR), NSE, CEA, CA19- 
Table 2 Clinical character of patients in different second-line therapy groups

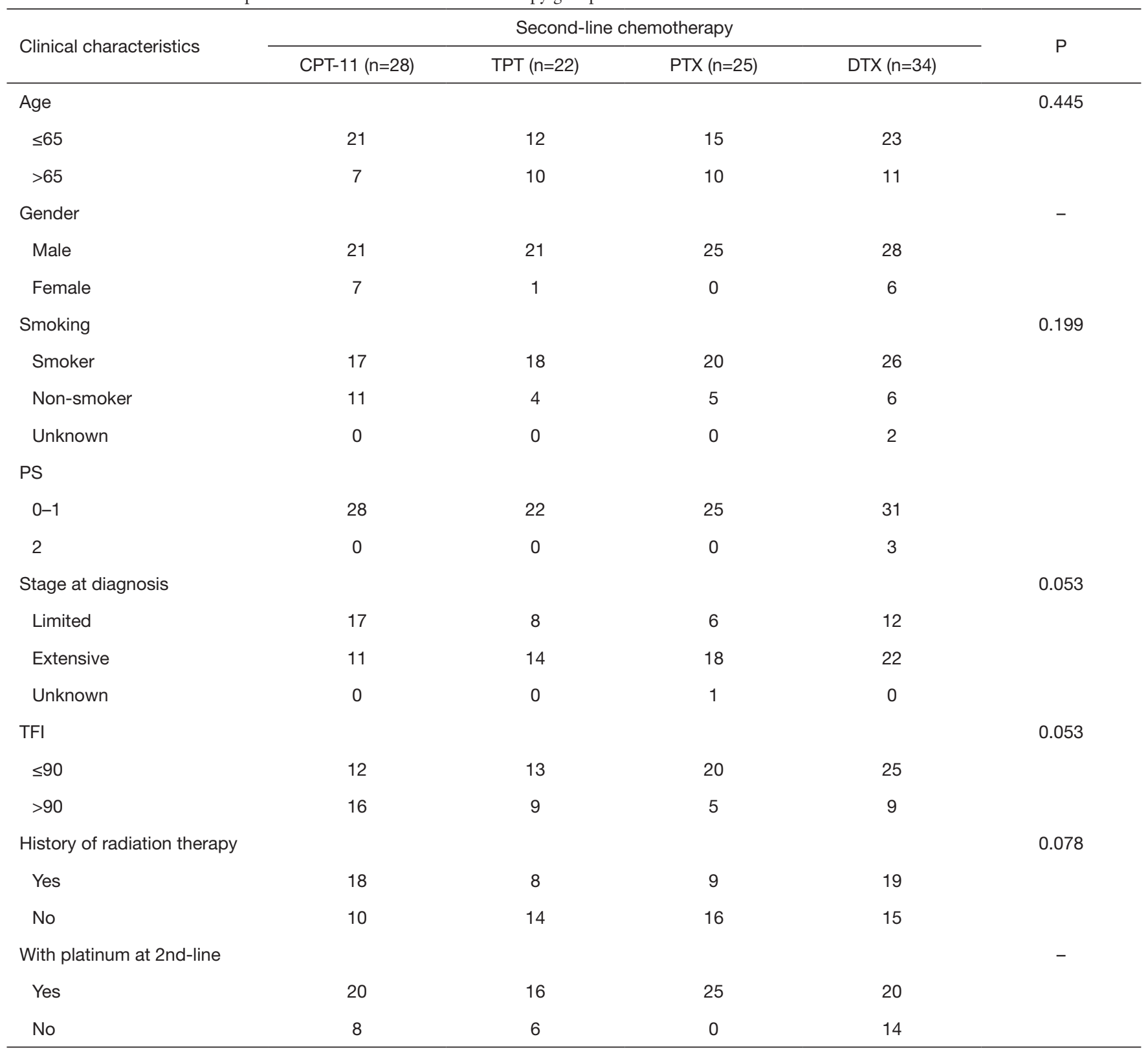

CPT-11, irinotecan; TPT, topotecan; PTX, paclitaxel; DTX, docetaxel; TFI, treatment free interval.

9 and CA125 were chosen as $300 \times 10^{9} / \mathrm{L}, 225 \mathrm{U} / \mathrm{L}, 3.5$, $16.3 \mathrm{ng} / \mathrm{mL}, 5 \mathrm{ng} / \mathrm{mL}, 40 \mathrm{ng} / \mathrm{mL}$ and $35 \mathrm{ng} / \mathrm{mL}$ respectively, based on the results of the evaluation of various cut-off values (13-19). Univariate analyses were performed by $\mathrm{K}-\mathrm{M}$ curves, revealed that the survival of SCLC patients was associated with five factors: stage $(\mathrm{P}=0.0089)$, TFI $(\mathrm{P}=0.0105)$, LDH $(\mathrm{P}=0.0105)$, NLR $(\mathrm{P}=0.016)$ and NSE levels $(\mathrm{P}=0.0209)$. No significant impact on survival was observed for the character of patients' gender, age, smoke state and past history of radiation therapy or the blood indicators of PLT, CEA, CA19-9 and CA125. After removing of the confounding factor of chemotherapy, multivariate analysis was performed by COX regression, results out that TFI $\geq 90$ days, $\mathrm{LDH} \geq 225 \mathrm{U} / \mathrm{L}$ and $\mathrm{NLR} \geq 3.5$ are independent prognostic factors for poor prognosis in patients received 


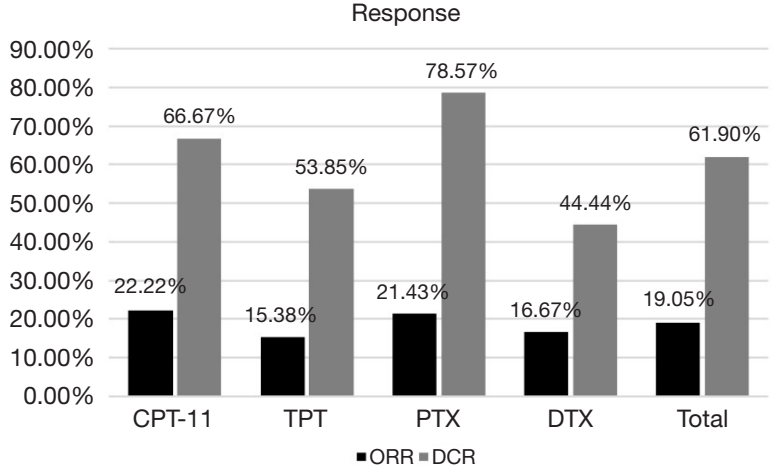

Figure 1 Response (DCR, ORR) in different second-line therapy groups. CPT-11, irinotecan; TPT, topotecan; PTX, paclitaxel; DTX, docetaxel; ORR, objective response rate; DCR, disease control rate.

Table 3 Correlation between response to first- and second-line chemotherapy

\begin{tabular}{lcccc}
\hline \multirow{2}{*}{$\begin{array}{l}\text { Response } \\
\text { to first-line }\end{array}$} & \multicolumn{4}{c}{ Response to second-line, n (\%) } \\
\cline { 2 - 5 } & $\mathrm{CR}$ & $\mathrm{PR}$ & $\mathrm{SD}$ & $\mathrm{PD}$ \\
\hline $\mathrm{CR}$ & $1(25.0)$ & $2(50.0)$ & $1(25.0)$ & 0 \\
$\mathrm{PR}$ & $1(5.3)$ & $4(21.1)$ & $7(36.8)$ & $7(36.8)$ \\
$\mathrm{SD}$ & 0 & $3(9.7)$ & $15(48.4)$ & $13(41.9)$ \\
$\mathrm{PD}$ & 0 & 0 & $3(42.9)$ & $4(57.1)$ \\
\hline
\end{tabular}

CR, complete response; PR, partial response; SD, stable disease; $P D$, progressive disease.

second-line chemotherapy.

\section{Discussion}

This retrospective study was conducted to evaluate clinical benefit of different second-line chemotherapy for SCLC patients in real clinical practice, then provide reference for clinical treatment. We analyzed 116 patients who received second-line chemotherapy with recurrence or progression after the first-line chemotherapy. These 116 patients were divided into 4 different groups according to the secondline chemotherapy they received: 28 in irinotecan (CPT-11) group, 22 in topotecan (TPT) group, 25 in paclitaxel (PTX) group and 34 in docetaxel (DTX) group. The clinical benefit was evaluated by the primary endpoints of PFS and OS, and the secondary endpoints of ORR and DCR.

As reported in the previous studies, the RR to secondline chemotherapy ranges a lot in people with different
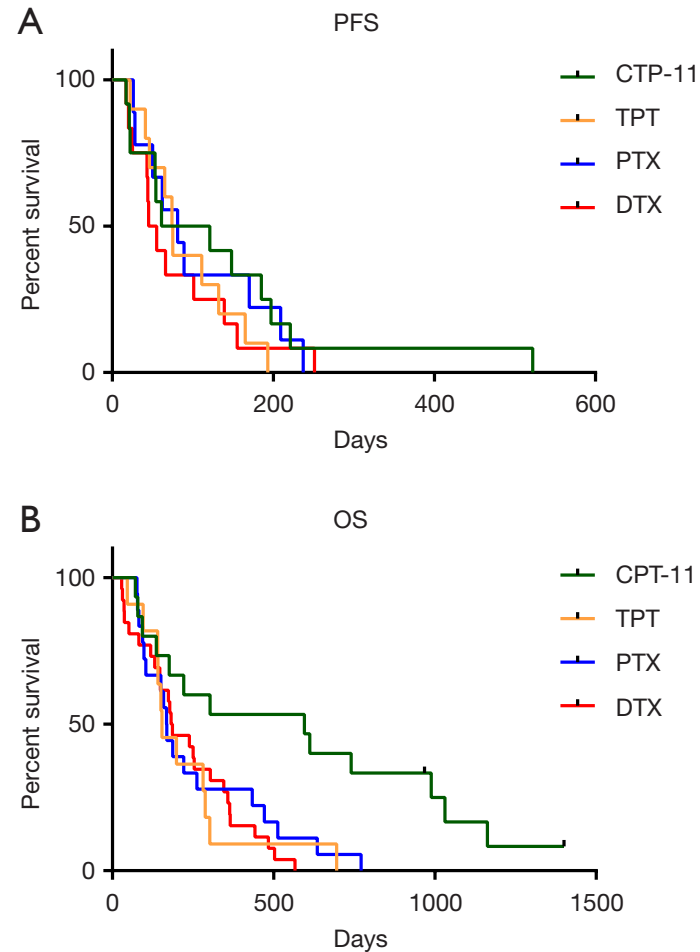

Figure 2 The survival analysis of different second-line therapy groups. (A) PFS in different second-line therapy groups. The medium PFS of CPT-11, TPT, PTX, DTX was 91, 74.5, 81, 50 days respectively. (B) OS in different second-line therapy groups. The medium OS of CPT11, TPT, PTX, DTX was 595, 154, 168.5, 184 days respectively. CPT11, irinotecan; TPT, topotecan; PTX, paclitaxel; DTX, docetaxel.

OS

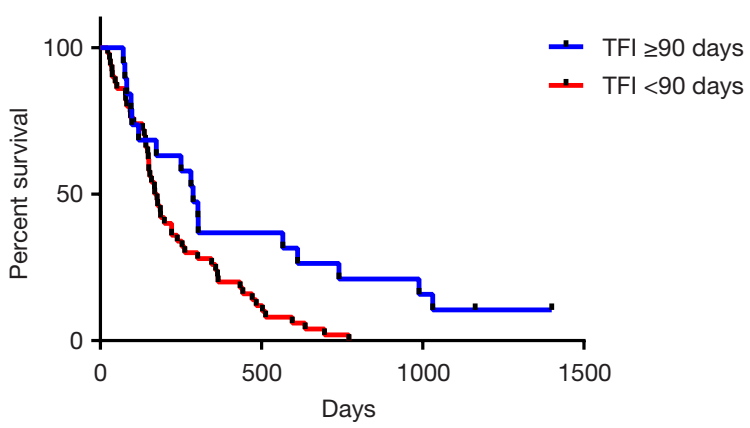

Figure 3 OS of patients with different TFI (TFI $\geq 90$ days and TFI $<90$ days). The medium OS of patients with TFI $\geq 90$ days and TFI $<90$ days was 288 and 172.5 days. TFI, treatment free interval.

types of recurrence or using different therapy. A metaanalysis reviewed 21 studies (20) showed that patients with sensitive recurrent achieved a higher ORR when compared 
Table 4 Univariate and multivariate analysis

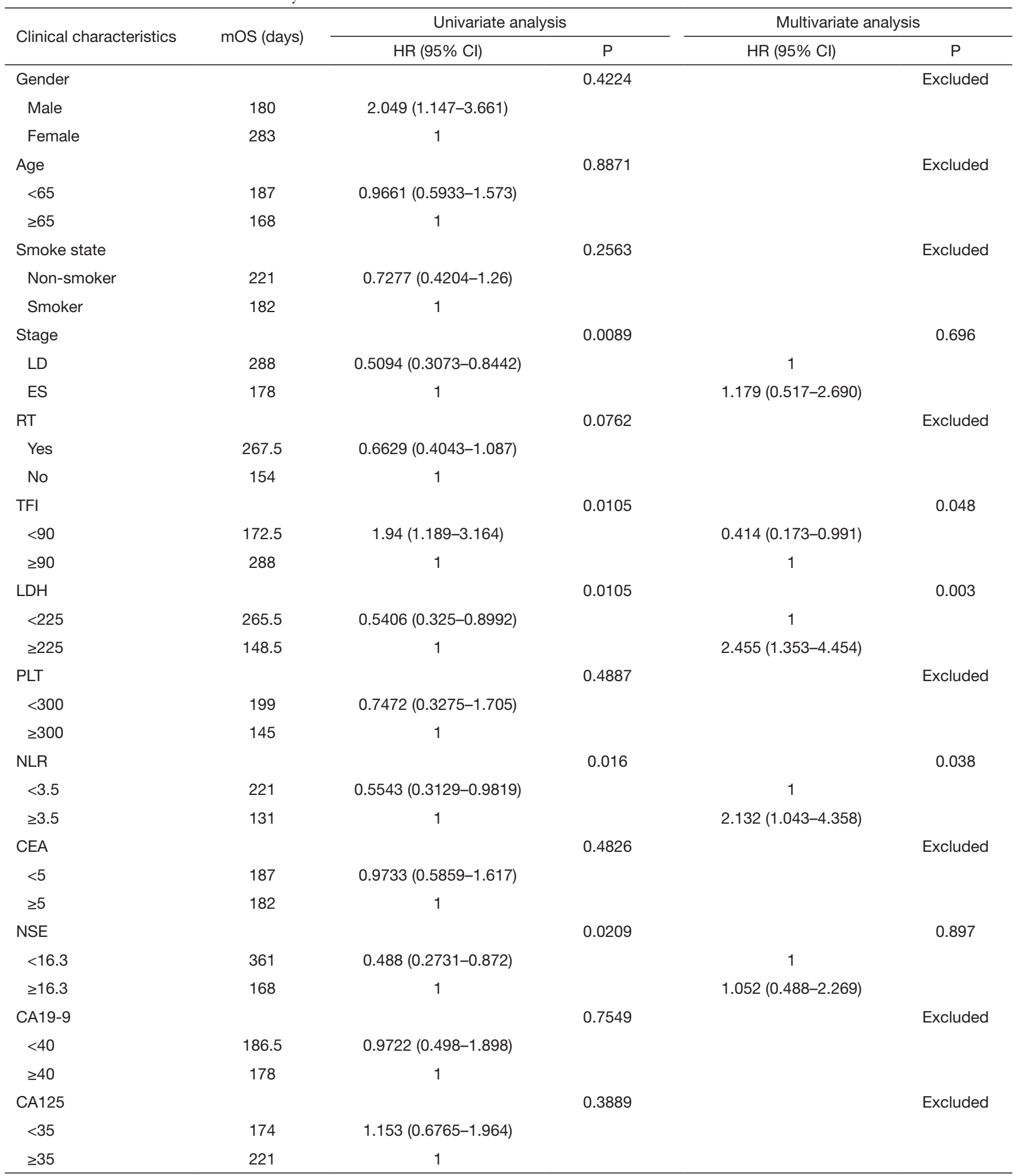

LD, limited disease; ES, extensive stage; TFI, treatment free interval; LDH, lactate dehydrogenase; PLT, platelet; NLR, neutrophil-tolymphocyte ratio; CEA, carcinoembryonic antigen; NSE, neurone specific enolase. 
to the refractory patients $(27.7 \%$ vs. $14.8 \%, \mathrm{P}=0.001)$. In our study, the overall ORR and DCR to second-line therapy was $19.05 \%$ and $61.90 \%$, and the medium PFS and OS was 75 days and 180 days. For different chemotherapy groups, PTX achieved the best DCR of $78.57 \%$, while CPT-11 achieved the best ORR of $22.22 \%$ (Figure 1). Patients treated with CPT-11 also achieved the best median PFS and OS of 91 and 595 days, while the median PFS of TPT, PTX and DTX were 74.5, 81 and 50 days respectively, the median OS of them were 154, 168.5 and 184 days respectively (Figure 2).

TPT is the only drug currently approved in Europe and the USA for sensitive relapsed SCLC. In an RCT study conducted in 2003, TPT showed significant survival advantage comparing to best supportive care (BSC, 6.4 vs. 3.4 months) (21). In the other phase III studies, the survival of TPT compared with CAV (cyclophosphamide, doxorubicin, and vincristine), amrubicin and CEI (cisplatin, etoposide, and irinotecan) did not show survival advantage. The medium OS of TPT in these studies ranges from 6.2 to 12.5 months (22-24). In our study, the medium OS of patients in TPT group was 154 days (5.1 months), the lowest among the four groups. The ORR of patients in TPT group was also the lowest, at $15.38 \%$. This may be partly related to the higher proportion of patients in this group of patients with extensive stage, which is $63.6 \%$.

CPT -11 is a topoisomerase I inhibitor which was recommended as second-line therapy of SCLC in NCCN SCLC guidelines. The combination of CEI improved the survival of advanced SCLC patients when compared with TPT (18.2 vs. 12.5 months) (24). However, the toxicity of this approach was significant, and it was not recommended by the guidelines. Another phase II trial compared the efficacy of CPT-11 monotherapy versus the combination of CPT-11 and gemcitabine as second-line treatment of patients with extensive stage SCLC. While the medium TTP of the 31 patients in CPT-11 monotherapy group was 1.7 months, the medium OS of them was 4.6 months (25). So far, there is no study comparing the efficiency of TPT and CPT-11 monotherapy for SCLC second-line therapy. The 33 patients who were treated with CPT-11 in our study got much longer mOS of 595 days (19.8 months). This might be partly because there were more patients in limit stage and had longer TTP in the CPT-11 group. The result needs to be validated in prospective trials which involve larger sample.

PTX used to be considered as promising agent to drugresistant SCLC. In a phase II study, PTX demonstrated a $29 \% \mathrm{RR}$ in refractory patients and $38 \%$ in sensitive patients (26). PTX is also believed to be able to enhance the antitumor activity of gemcitabine in NSCLC patients (27), and the lack of cross-resistance between PTX and cisplatin was confirmed in human SCLC lines (28). A phase II evaluated the efficiency of PTX and gemcitabine combination as second-line chemotherapy in 41 SCLC patients, which contains 19 with refractory disease and 22 with sensitive disease, results out that $22 \%$ of the patients achieved PR and $46 \%$ achieved disease control. The medium OS was 5.5 months in this study (29). In our study, the 25 patients in PTX group were all treated with platinum in combination. They were more responsive to the treatment with the DCR of $78.57 \%$ and has a relatively longer PFS of 81 days. However, the advantage on the OS was not seen in this group, which was 168.5 days.

Little research was conducted on the use of DOX in second-line therapy of SCLC. There was a phase II study involving 34 patients with previous treated SCLC reported that $25 \%$ of 28 evaluable patients achieved PR in their trial (30). In our study, the DOX group was much less sensitive to the chemotherapy than the others, the DCR and ORR in this group was $44.44 \%$ and $16.67 \%$, and the mPFS was the shortest in four groups, which was 50 days. But the mOS of this group was not worse than the TPT and PTX group, which was 180 days.

The efficacy of second-line chemotherapy of is highly dependent on the time from initial therapy to relapse. Huisman (31) and Glisson (32) have reported that patients with SCLC who have not benefited from the first-line therapy may have lower second-line benefits. In our study, the correlation analysis of response to first-line and secondline treatment also showed that patients who reacted good to first-line treatment would be more likely to obtain benefit from second-line treatment (Table 3).

The duration and quality of response to first-line therapy was also reported to be strongly predict the response and survival outcome in second-line therapy. A lot of studies have confirmed that patient with sensitive recurrent have longer OS than the refractory patients $(12,20,33)$. In this study, we furtherly clarified which factor is concerned about the second-line therapy OS of SCLC. We classified patients into two groups according to their time to progress from the first-line therapy, find out that patients whose TFI was longer than 90 days had significantly longer OS than whose TFI was shorter (9.6 vs. 5.75 months, Figure 3). Other clinical character and blood index were also included into the prognosis analysis, and the univariate analysis showed that ES-stage, TFI $<90$ days, $\mathrm{LDH} \geq 225 \mathrm{U} / \mathrm{L}, \mathrm{NLR} \geq 3.5$, 
NSE $\geq 16.3 \mathrm{ng} / \mathrm{mL}$ were associated with poor prognosis. After removing of the confounding factor of chemotherapy, TFI $<90$ days, $\mathrm{LDH} \geq 225 \mathrm{U} / \mathrm{L}$ and NLR $\geq 3.5$ were identified as independent risk factors for second-line SCLC patients in multivariate analysis (Table 4).

Although this study has the limitation of single-center retrospective study and the defect of the heterogeneity on the characteristics and management of the patients evaluated, it also has its advantages, such as the consistency of the source of the patient, the implementation of the treatment plan and the evaluation of the efficacy. It provides comparation in the real-world to the efficacy of four different second-line chemotherapy of SCLC and reference for clinical selection of second-line chemotherapy. This study also identified TFI $<90$ days, $\mathrm{LDH} \geq 225 \mathrm{U} / \mathrm{L}$ and NLR $\geq 3.5$, as independent risk factors for second-line SCLC patients, which might assist in clinical risk profile, and they might be worth considering as stratification factors when selecting candidates for secondline chemotherapy in future clinical trials.

\section{Acknowledgments}

Funding: This work was supported by grants from the National Natural Science Foundation of China (grant number 81671892, 81401903, 81572937 and 81572273); the Jiangsu Provincial Special Program of Medical Science (BE2018743); the 16th batch "Summit of the Six Top Talents" Program of Jiangsu Province (grant number WSN-154); China Postdoctoral Science Foundation 12th batch Special fund (Postdoctoral number: 45786); China Postdoctoral Science Foundation 64th batch (Postdoctoral number: 45786); Jiangsu Provincial Postdoctoral Science Foundation (grant number 2018K049A); the Natural Science Foundation of Jiangsu province (grant number BK20180139 and BK20161386); Jiangsu Provincial Medical Youth Talent (grant number QNRC2016125), and the Nanjing Medical Science and Technology Development Project (No. ZKX17044), the Jiangsu Provincial Key Research and Development Program (No. BE2016721).

\section{Footnote}

Conflicts of Interest: The authors have no conflicts of interest to declare.

Ethical Statement: The authors are accountable for all aspects of the work in ensuring that questions related to the accuracy or integrity of any part of the work are appropriately investigated and resolved. This study is a retrospective study, approved by the ethics committee of Nanjing Jinling Hospital (No. 2017NZGKJ-128), without the need for informed consent.

\section{References}

1. Lally BE, Urbanic JJ, Blackstock AW, et al. Small cell lung cancer: have we made any progress over the last 25 years? Oncologist 2007;12:1096-104.

2. Schneider BJ. Management of recurrent small cell lung cancer. J Natl Compr Canc Netw 2008;6:323-31.

3. Hansen HH. Management of small-cell cancer of the lung. Lancet 1992;339:846-9.

4. Hamilton G, Rath B. Targeting angiogenesis in small cell lung cancer. Transl Cancer Res 2017;6:S522-8.

5. Farago AF, Keane FK. Current standards for clinical management of small cell lung cancer. Transl Lung Cancer Res 2018;7:69-79.

6. Ando M, Kobayashi K, Yoshimura A, et al. Weekly administration of irinotecan (CPT-11) plus cisplatin for refractory or relapsed small cell lung cancer. Lung Cancer 2004;44:121-7.

7. Groen HJ, Fokkema E, Biesma B, et al. Paclitaxel and carboplatin in the treatment of small-cell lung cancer patients resistant to cyclophosphamide, doxorubicin, and etoposide: a non-cross-resistant schedule. J Clin Oncol 1999;17:927-32.

8. Inoue A, Sugawara S, Yamazaki K, et al. Randomized phase II trial comparing amrubicin with topotecan in patients with previously treated small-cell lung cancer: North Japan Lung Cancer Study Group Trial 0402. J Clin Oncol 2008;26:5401-6.

9. Masuda N, Fukuoka M, Kusunoki Y, et al. CPT-11: a new derivative of camptothecin for the treatment of refractory or relapsed small-cell lung cancer. J Clin Oncol 1992;10:1225-9.

10. Allen JW, Moon J, Redman M, et al. Southwest Oncology Group S0802: a randomized, phase II trial of weekly topotecan with and without ziv-aflibercept in patients with platinum-treated small-cell lung cancer. J Clin Oncol 2014;32:2463-70.

11. Janjigian YY, Bendell J, Calvo E, et al. CheckMate-032 Study: Efficacy and Safety of Nivolumab and Nivolumab Plus Ipilimumab in Patients With Metastatic Esophagogastric Cancer. J Clin Oncol 2018;36:2836-44.

12. Postmus PE, Berendsen HH, van Zandwijk N, et al. Retreatment with the induction regimen in small cell lung 
cancer relapsing after an initial response to short term chemotherapy. Eur J Cancer Clin Oncol 1987;23:1409-11.

13. Zhang H, Zhang L, Zhu K, et al. Prognostic Significance of Combination of Preoperative Platelet Count and Neutrophil-Lymphocyte Ratio (COP-NLR) in Patients with Non-Small Cell Lung Cancer: Based on a Large Cohort Study. PLoS One 2015;10:e0126496.

14. Liu ZL, Zeng TT, Zhou XJ, et al. Neutrophil-lymphocyte ratio as a prognostic marker for chemotherapy in advanced lung cancer. Int J Biol Markers 2016;31:e395-401.

15. Shen L, Zhang H, Liang L, et al. Baseline neutrophillymphocyte ratio $(>/=2.8)$ as a prognostic factor for patients with locally advanced rectal cancer undergoing neoadjuvant chemoradiation. Radiat Oncol 2014;9:295.

16. McIntyre LM, Thorburn DR, Bubb WA, et al. Comparison of computer simulations of the F-type and L-type non-oxidative hexose monophosphate shunts with 31P-NMR experimental data from human erythrocytes. Eur J Biochem 1989;180:399-420.

17. Bremnes RM, Sundstrom S, Aasebo U, et al. The value of prognostic factors in small cell lung cancer: results from a randomised multicenter study with minimum 5 year follow-up. Lung Cancer 2003;39:303-13.

18. Isaksson S, Jonsson P, Monsef N, et al. CA 19-9 and CA 125 as potential predictors of disease recurrence in resectable lung adenocarcinoma. PLoS One 2017;12:e0186284.

19. Flanders WD, Lally CA, Zhu BP, et al. Lung cancer mortality in relation to age, duration of smoking, and daily cigarette consumption: results from Cancer Prevention Study II. Cancer Res 2003;63:6556-62.

20. Owonikoko TK, Behera M, Chen Z, et al. A systematic analysis of efficacy of second-line chemotherapy in sensitive and refractory small-cell lung cancer. J Thorac Oncol 2012;7:866-72.

21. O'Brien ME, Ciuleanu TE, Tsekov H, et al. Phase III trial comparing supportive care alone with supportive care with oral topotecan in patients with relapsed small-cell lung cancer. J Clin Oncol 2006;24:5441-7.

22. von Pawel J, Schiller JH, Shepherd FA, et al. Topotecan versus cyclophosphamide, doxorubicin, and vincristine for the treatment of recurrent small-cell lung cancer. J Clin Oncol 1999; 17:658-67.

23. von Pawel J, Jotte R, Spigel DR, et al. Randomized phase III trial of amrubicin versus topotecan as second-line treatment for patients with small-cell lung cancer. J Clin Oncol 2014;32:4012-9.

24. Goto K, Ohe Y, Shibata T, et al. Combined chemotherapy with cisplatin, etoposide, and irinotecan versus topotecan alone as second-line treatment for patients with sensitive relapsed small-cell lung cancer (JCOG0605): a multicentre, open-label, randomised phase 3 trial. Lancet Oncol 2016;17:1147-57.

25. Pallis AG, Agelidou A, Agelaki S, et al. A multicenter randomized phase II study of the irinotecan/gemcitabine doublet versus irinotecan monotherapy in previously treated patients with extensive stage small-cell lung cancer. Lung Cancer 2009;65:187-91.

26. Smit EF, Fokkema E, Biesma B, et al. A phase II study of paclitaxel in heavily pretreated patients with small-cell lung cancer. Br J Cancer 1998;77:347-51.

27. Kroep JR, Giaccone G, Voorn DA, et al. Gemcitabine and paclitaxel: pharmacokinetic and pharmacodynamic interactions in patients with non-small-cell lung cancer. J Clin Oncol 1999;17:2190-7.

28. Jensen PB, Holm B, Sorensen M, et al. In vitro crossresistance and collateral sensitivity in seven resistant smallcell lung cancer cell lines: preclinical identification of suitable drug partners to taxotere, taxol, topotecan and gemcitabin. Br J Cancer 1997;75:869-77.

29. Dazzi C, Cariello A, Casanova C, et al. Gemcitabine and paclitaxel combination as second-line chemotherapy in patients with small-cell lung cancer: a phase II study. Clin Lung Cancer 2013;14:28-33.

30. Smyth JF, Smith IE, Sessa C, et al. Activity of docetaxel (Taxotere) in small cell lung cancer. The Early Clinical Trials Group of the EORTC. Eur J Cancer 1994;30A:1058-60.

31. Huisman C, Postmus PE, Giaccone G, et al. Second-line chemotherapy and its evaluation in small cell lung cancer. Cancer Treat Rev 1999;25:199-206.

32. Glisson BS. Recurrent small cell lung cancer: update. Semin Oncol 2003;30:72-8.

33. Jett JR, Schild SE, Kesler KA, et al. Treatment of small cell lung cancer: Diagnosis and management of lung cancer, 3rd ed: American College of Chest Physicians evidence-based clinical practice guidelines. Chest 2013;143:e400S-e419S.

Cite this article as: Zhao Y, Wan B, Zhang T, Xu Y, Liu H, Lv T, Zhang F, Zhan P, Song Y. Irinotecan, topotecan, paclitaxel or docetaxel for second-line treatment of small cell lung cancer: a single-center retrospective study of efficiency comparation and prognosis analysis. Transl Lung Cancer Res 2019;8(6):829-837. doi: $10.21037 /$ tlcr.2019.10.21 